\title{
Distributed Control with Integral Quadratic Constraints
}

\author{
Hui Fang and Panos J. Antsaklis
}

\begin{abstract}
In this paper, stability conditions for distributed control problems are derived under general integral quadratic constraints to achieve quadratic performance. These results take the form of coupled LMIs, and the multipliers are specified by the underlying integral quadratic constraints to model interconnections between the subsystems. It is further shown that these stability results can be exploited for distributed controller synthesis in a similar way to the gain-scheduling controller design in the LPV systems. The main contribution of this paper is to unify previous stability results in one general framework of Integral Quadratic Constraints (IQC) analysis and provide lower dimension controller synthesis conditions.
\end{abstract}

\section{INTRODUCTION}

Over the past few years, there has been renewed research interest in distributed control of large scale systems; see for example, [1],[2], [3], [4], [5], [6], [7], [8], [9]. These systems are formed by the interconnection of multiple homogeneous or heterogeneous subsystems. Their overall complex dynamical behavior is dictated by their distributed nature and the dynamical interactions between the subsystems.

The spatially distributed nature of the system and the presence of interconnections make the sharing of feedback information challenging. This factor has motivated new research directions in control theory where communication constraints are considered explicitly. In particular, researchers have considered control problems with non-ideal communication links such as limited bandwidth [10], [11], delay, and packet dropout between sensors and actuator of these subsystems.

Because of communication and computation constraints, it is often useful to take advantage of special structures of the underlying topology of the systems. Successful synthesis methods have been proposed for the existence of decentralized controllers guaranteeing performance. However, these techniques only apply to systems with specialized interconnections. For a collection of recent research results in this area, see [12], [13] and the references therein.

Recently, a distributed control theory was developed for spatially-invariant distributed systems [2]. It is shown that the controllers have 'identical' structure as the underlying subsystems. A linear matrix inequality (LMI) based control synthesis algorithm for this class of interconnected systems was developed in [2], [3] using a multidimensional 
system theory. These results were further extended in [1], [5], [14] to distributed systems over an arbitrary graph under various communication conditions. Specifically, the results take the form of a set of coupled linear matrix inequalities, where the particular design variables for the LMIs are shaped by the interconnections. It should be pointed out that these stability results can be explained in the general framework of dissipativity theory [15], and are related to the integral quadratic constraints (IQC) [16] analysis methods, since the interconnections are generally modeled as IQCs. This distributed control theory has recently been applied to the control of large-scale irrigation networks in Australia [17] and the performance is compared to standard centralized and decentralized control techniques. It was shown that distributed controllers achieved similar performance to centralized controllers and significantly better error propagation performance than simple decentralized feedback controllers. Since the computational cost and the infrastructure cost for decentralized control does not scale well with the number of subsystems, the distributed control appears to achieve an acceptable trade-off between performance and complexity.

The objective of this paper is first to characterize the stability conditions for distributed systems with IQC constraints, and then to introduce a distributed controller design method using topological structures and the property of the interconnections. The main contribution of our approach is to unify the stability results that first appeared in [1] in one general framework of IQC analysis. This analysis is similar to what was proposed in [18], [19]. However, here there is much more emphasis on the communication constraints between subsystems and the decoupled stability conditions. Although stability conditions are available for the global system when it is seen as a single system, these results are not directly applicable to distributed control design. In this paper, we show that distributed stability conditions exist for some IQCs that can be modeled by a set of multipliers. These distributed stability conditions can be later utilized for distributed controller synthesis. Another important contribution of the present paper is to relate distributed control under communication constraints to the well-developed results in the literature of gain-scheduling techniques for linear parameter varying (LPV) systems [19], [18]. Establishing and explaining the relations between distributed control and LPV is important as it opens the way for using in distributed control problems the results from the well-established field of LPV. Geometrically, the stability results can be interpreted from a graph separation point view ([20], [21] [22]) following a similar proof as in [19]. As for synthesis, based on a recently extended elimination lemma in [23], the synthesis inequalities turn out to be convex in all variables, including the scalings [24]. It is also worth mentioning that in [1] the dimension of the controller $n_{i j}^{K}=3 n_{i j}$ for the synthesis condition, while here we show that if the dimension of the distributed controller is greater than or equal to the associated interconnected signals for the plants, i.e, $n_{i j}^{K} \geq n_{i j}$, there exist distributed controllers to guarantee the global control performance.

The paper is organized as follows: We begin with some mathematical preliminaries and in section II, the distributed system models are introduced. Section III is devoted to analysis of stability and performance of the distributed systems under various interconnections. In Section IV, we derive distributed controller synthesis results and some concluding remarks are made in Section V.

Notation. The set of real number is denoted by $\mathbf{R}$, the nonnegative reals by $\mathbf{R}^{+} . \mathbf{R}^{n \times m}$ is the set of $n \times m$ matrices. The transpose(complex conjugate transpose) of matrix $M$ is denoted by $M^{T}\left(M^{*}\right)$. We use $\mathbf{R}_{S}^{n}$ to denote $n \times n$ real 
symmetric matrices. If $M \in \mathbf{R}_{S}^{n}$, then $M>0(M \geq 0)$ indicate $M$ is positive definite (positive semidefinite) matrix, and $M<0(M \leq)$ denotes negative (negative semidefinite) matrix. For any matrix $P$, $\operatorname{ker}(P)$ stands for the null space of the linear operator associated with $P$. The inertia of a symmetric matrix $A$ is the ordered triple in $(A)=$ $\left(i_{+}(A), i_{0}(A), i_{-}(A)\right)$ where $i_{+}(A), i_{-}(A), i_{0}(A)$ is the number of positive, negative and zero eigenvalues of $A$, all counting multiplicity. A block diagonal matrix with $X_{k}, \ldots, X_{l}$ is denoted $\operatorname{diag}_{k \leq i \leq l} X_{i}=\operatorname{diag}\left\{X_{k}, \ldots, X_{l}\right\}$; if $e_{1}, \ldots, e_{L}$ are elements of sets $E_{1}, \ldots, E_{L}, \operatorname{cat}_{k \leq i \leq l} e_{i}$ will designate the elements $\left(e_{k}, \ldots, e_{l}\right) \in E_{k} \times \ldots E_{l}$ when $1 \leq k \leq l \leq L$. We will sometimes write $\operatorname{diag}_{i}$ and cat $_{i}$ instead of $\operatorname{diag}_{1 \leq i \leq L}$ and cat $_{1 \leq i \leq L}$.

The Euclidean norm of a vector $x \in \mathbf{R}^{n}$ is denoted by $\|x\|=\left(x^{T} x\right)^{1 / 2}$. The space of square integrable $n$ dimensional functions $f:(0, \infty) \rightarrow \mathbf{R}^{n}$ is denoted by $\mathcal{L}_{2}^{n}$; this is abbreviated as $\mathcal{L}_{2}$ when $n$ is clear from context or not relevant. The Fourier transform of a $\mathcal{L}_{2}$ function $f$ is denoted as $\hat{f}(j \omega)$. The norm of an $\mathcal{L}_{2}$ signal and the induced norm of an operator on $\mathcal{L}_{2}$ is denoted by $\|\cdot\|$, so for an operator $F: \mathcal{L}_{2} \rightarrow \mathcal{L}_{2},\|F\|=\sup _{u \in \mathcal{L}_{2}}\|F u\|$. An operator $\Delta: \mathcal{L}_{2}^{n} \rightarrow \mathcal{L}_{2}^{n}$ is said to be contractive if $\|\Delta v\| \leq\|v\|, \forall v \in \mathcal{L}_{2}^{n}$. Lower case $\delta$ 's always denote operators from $\mathcal{L}_{2}^{1}$ to $\mathcal{L}_{2}^{1}$, for $u, v \in \mathcal{L}_{2}^{n}$, the express $v=\delta I_{n} u$ is defined to mean that $u_{k}$ of $u$ and $v_{k}$ of $v$ satisfy $u_{k}=\delta v_{k}$. An operator $\delta: \mathcal{L}_{2} \rightarrow \mathcal{L}_{2}$ is called self-adjoint if $\langle u, \delta v\rangle=\langle\delta u, v\rangle, \forall u, v \in \mathcal{L}_{2}$. Note that all real-valued static LTV operators are self-adjoint.

\section{PRoblem Formulation}

\section{A. Problem Formulation}

In this paper, we will concern ourselves with systems formulated as follows. The global system consists of an assembly of $L$ subsystem $G_{i}, i=1, \ldots, L$ connected arbitrarily. Each subsystems $G_{i}$ is captured by the following state-space equations:

$$
\begin{aligned}
{\left[\begin{array}{c}
\dot{x}_{i}(t) \\
w_{i}(t) \\
z_{i}(t)
\end{array}\right] } & =\left[\begin{array}{ccc}
A_{T T}^{i} & A_{T S}^{i} & B_{T}^{i} \\
A_{S T}^{i} & A_{S S}^{i} & B_{S}^{i} \\
C_{T}^{i} & C_{S}^{i} & D^{i}
\end{array}\right]\left[\begin{array}{c}
x_{i}(t) \\
v_{i}(t) \\
d_{i}(t)
\end{array}\right] \\
x_{i}(0) & =x_{i}^{0}
\end{aligned}
$$

where $x_{i}(t) \in \mathbf{R}^{m_{i}}, d_{i}(t) \in \mathbf{R}^{p_{i}}, z_{i}(t) \in \mathbf{R}^{q_{i}}, v_{i}(t), w_{i}(t) \in \mathbf{R}^{n_{i}}$ for all $t \geq 0$. In (1), $d_{i}$ is the disturbance and $z_{i}$ is the performance associated with $G_{i}$, while $v_{i}$ and $w_{i}$ are the overall interconnection signals used by $G_{i}$. For each given $i, v_{i}, w_{i}$ is further partitioned into $v_{i j}, w_{i j}$ respectively. $v_{i j}, w_{i j}$ are $n_{i j}$-dimension interconnection signals for subsystems $G_{i}, G_{j}$. We use an operator $\Delta_{i j}$ to model the input-output relationship for the interconnection between subsystem $G_{i}$ and $G_{j}$, such that,

$$
v_{i j}=\Delta_{j i} w_{j i}, \quad \forall i, j, 1 \leq i, j \leq L
$$

In this model, $w_{j i}$ is the input signal and $v_{i j}$ is the output signal for the communication channel from $G_{j}$ to $G_{i}$; on the other hand, $w_{j i}$ is an output signal for subsystem $G_{j}$, transmitted from subsystem $G_{j}$ to subsystem $G_{i}$ and and can be regarded as an input signal $v_{i j}$ for subsystem $G_{i}$. For example, the simplest case could be, $w_{j i}=v_{i j}$ which is called perfect interconnection since the input signal $w_{j i}$ transmitted from $G_{j}$ to $G_{i}$ is perfectly recovered. 
The signals considered in this paper are square integrable, i.e, $\mathcal{L}_{2}$. Let $\Delta_{i j}: \mathcal{L}_{2} \rightarrow \mathcal{L}_{2}$ be an operator to model the interconnection between subsystem $i$ and $j$. The signal subspace defined by $\Delta_{i j}$ is $\mathcal{W}\left(\Delta_{i j}\right)$,

$$
\mathcal{W}\left(\Delta_{j i}\right)=\left\{\left[\begin{array}{c}
v_{i j} \\
w_{j i}
\end{array}\right] \in \mathcal{L}_{2}^{2 n_{j i}}: v_{i j}=\Delta_{j i} w_{j i}\right\}
$$

If we denote $v=\operatorname{cat}_{i} v_{i}$, where each $v_{i}$ can be further partitioned as $v_{i}=\mathbf{c a t}_{j} v_{i j}$. For the global system, $x=\mathbf{c a t}_{i} x_{i}, w=\mathbf{c a t}_{i} \mathbf{c a t}_{j} w_{i j}, z=\mathbf{c a t}_{i} z_{i}, d=\mathbf{c a t}_{i} d_{i}$ are similarly defined. Note that the dimension of $v_{i j}, v_{i}$ and $v$ are $n_{i j}, n_{i}$ and $\mathcal{N}$ where $n_{i}=\sum_{j=1}^{L} n_{i j}, \mathcal{N}=\sum_{i=1}^{L} n_{i}$.

Based on the state space representations of $G_{i}$, the state space representation of the global system can be described as follows:

$$
\begin{aligned}
{\left[\begin{array}{c}
\dot{x}(t) \\
w(t) \\
z(t)
\end{array}\right] } & =\left[\begin{array}{c|cc}
A_{T T} & B_{T S} & B_{T d} \\
\hline A_{S T} & A_{S S} & B_{S d} \\
C_{T z} & C_{S z} & D_{z d}
\end{array}\right]\left[\begin{array}{c}
x(t) \\
v(t) \\
d(t)
\end{array}\right] \\
v(t) & =\Delta P w(t)
\end{aligned}
$$

where $\Delta \in \Delta$ is a (causal) operator from $\mathcal{L}_{2}^{\mathcal{N}}$ to $\mathcal{L}_{2}^{\mathcal{N}}$ generated via $\Delta_{i j}$,

$$
\Delta=\operatorname{diag}_{i} \operatorname{diag}_{j} \Delta_{j i}
$$

and the permutation matrix are chosen such that

$$
\bar{w}=\operatorname{cat}_{i} \mathbf{c a t}_{j} w_{j i}=P w
$$

matrix $A_{T T}=\operatorname{diag}_{i} A_{T T}^{i}$, all other matrices in (5) are diagonal matrices. Note that, although the system matrices and the operator $\Delta$ for the global system have a diagonal structure, the subsystems are coupled by the permutation matrix $P$ in (6) for interconnection signals $v$ and $w$. The signal space for for $v, w$ can be described as

$$
\mathcal{W}(\Delta)=\left\{\left[\begin{array}{c}
v \\
w
\end{array}\right] \in \mathcal{L}_{2}^{2 \mathcal{N}}:\left[\begin{array}{c}
v_{i j} \\
w_{j i}
\end{array}\right] \in \mathcal{L}_{2}^{2 n_{j i}}, v_{i j}=\Delta_{j i} w_{j i}\right\}
$$

For the state-space representation of the global system (5), we represent its transfer function as follows:

$$
\mathbf{G}=\left[\begin{array}{ll}
G_{11} & G_{12} \\
G_{21} & G_{22}
\end{array}\right]
$$

which has been partitioned to conform with the vector $(v, d)$.

In this paper, if the system (5) is internally stable independently of the uncertainty of the interconnections $\Delta_{i j}$ (9), it is defined as well-posed and stable.

Definition 2.1: The interconnected system consisting of subsystems (5) and the interconnection constraints (9) is said to be well-posed and stable if the map $\left(I-\Delta P G_{11}\right)$ has a bounded inverse on $\mathcal{L}_{2}$, for any choice of $\Delta$ specified.

Finally, we will say that the global system $\mathbf{G}$ is contractive if it is stable and $\|z\|<\|d\|$ for all $d \in \mathcal{L}_{2}$ and all interconnection $\Delta_{i j}$. 


\section{Stability Analysis for Distributed Systems}

The main idea here is to first use Integral Quadratic Constraints (IQC) to model the interconnection operator $\Delta_{i j}$. The performance under the integral quadratic constraints for the internal signal $v, w$ can then be casted as an unconstrained quadratic optimization problem. For the LTI system, the stability results admit a LMI formulation.

Generally speaking, the stability results for the global system can not be used directly for distributed controller design. Based on these results, we shall utilize the structure information dictated by the permutation matrix $P$ for distributed controller synthesis. By restricting our analysis to a particular set of multipliers for the IQCs, we can derive distributed stability conditions for each of the subsystems to guarantee global performance.

We will need the following definition of Integral Quadratic Constraints.

\section{A. Stability Analysis for the Global System}

Integral Quadratic Constraints (IQC) give useful characterizations of the structure of given operators. The IQCs are defined in term of quadratic forms which are defined in terms of self-adjoint operators. We will be particularly interested in the case when the operators are defined on the extended spaces $\mathcal{H}_{e}=\mathcal{L}_{2 e}^{m}[0, \infty)$. It is important to notice that IQC is defined on the Hilbert space $\mathcal{H}$.

Definition 3.1: [16] Let $\Pi$ be a bounded and self-adjoint operator. Then $\Delta$ satisfies the IQC defined by $\Pi$ if

$$
\sigma_{\Pi}(v, \Delta(v))=<\left[\begin{array}{c}
v \\
\Delta(v)
\end{array}\right], \Pi\left[\begin{array}{c}
v \\
\Delta(v)
\end{array}\right]>\geq 0, \quad \forall v \in \mathcal{H}
$$

We often call $\Pi$ the multiplier that defines IQC. We will sometimes use the shorthand notation $\Delta \in I Q C(\Pi)$ to mean that $\Delta$ satisfies the IQC defined by $\Pi$.

Depending on the particular application, both the frequency-domain and time-domain versions of IQCs are available. For example, if $\mathcal{H}=\mathcal{L}_{2}^{m}[0, \infty)$, then $\Pi$ can be taken as a transfer function satisfying $\Pi(j \omega)=\Pi(j \omega)^{*}$. The condition in (11) reduces to

$$
\sigma_{\Pi}(v, \Delta(v))=\int_{-\infty}^{\infty}\left[\begin{array}{c}
\hat{v} \\
\hat{\Delta}(v)
\end{array}\right]^{*} \Pi\left[\begin{array}{c}
\hat{v} \\
\hat{\Delta}(v)
\end{array}\right] d \omega \geq 0, \quad \forall v \in \mathcal{L}_{2}^{m}[0, \infty)
$$

Definition 3.2: Let $X, Y$ and $Z$ be constant real matrices and let the full rank matrix

$$
\Phi=\left[\begin{array}{cc}
X & Y \\
Y^{T} & Z
\end{array}\right]
$$

Let $H: \mathcal{L}_{2 e} \rightarrow \mathcal{L}_{2 e}$ be an operator. We say $H$ is $\{X, Y, Z\}$-dissipative if for every $p, q \in \mathcal{L}_{2 e}, p=H(q)$ implies that

$$
\int_{0}^{T}\left[\begin{array}{l}
p(t) \\
q(t)
\end{array}\right]^{T}\left[\begin{array}{cc}
X & Y \\
Y^{T} & Z
\end{array}\right]\left[\begin{array}{c}
p(t) \\
q(t)
\end{array}\right] d t \geq 0, \quad \forall T \geq 0
$$

Note that, condition (13) can be represented as a frequency-domain IQC (12) when $H$ is stable. For a stable operator $H$, we can recover a special kind of time-domain IQC.Precisely, the condition

$$
\int_{0}^{\infty}\left[\begin{array}{l}
p(t) \\
q(t)
\end{array}\right]^{T}\left[\begin{array}{cc}
X & Y \\
Y^{T} & Z
\end{array}\right]\left[\begin{array}{l}
p(t) \\
q(t)
\end{array}\right] d t \geq 0
$$


is equivalently to condition (13).

Many important properties of basic system interconnections used in stability analysis can be characterized by IQC's with proper multiplier П. A simple example of multiplier is

$$
\Pi_{1}=\left[\begin{array}{cc}
I & 0 \\
0 & -I
\end{array}\right]
$$

where $\Pi_{1}$ defines a valid IQC for operators that have gain less than one. A collection of common used IQC's has been summarized in [16].

Based on results on $(D, G)$-scaling [25], the following linear time varying operators of fixed block and scalar operators can be equivalently represented by IQCs with proper constant multiplier П's.

Lemma 1: - Suppose $\widetilde{\delta}: \mathcal{L}_{n}^{2} \rightarrow \mathcal{L}_{n}^{2}$, if the LTV operator $\widetilde{\delta}$ is self-adjoint and contractive, then for any $D \in \mathbf{R}_{S}^{n \times n}, D \geq 0$ and $G=-G^{T}, \widetilde{\delta} I_{n}$ is $(D, G,-D)$-dissipative.

- Suppose $\delta: \mathcal{L}_{n}^{2} \rightarrow \mathcal{L}_{n}^{2}$, if the LTV operator is contractive, then for any $D \in \mathbf{R}_{S}^{n \times n}, D \geq 0, \delta I_{n}$ is $(D, 0,-D)$ dissipative.

- There is a contractive LTV operator, $\Delta: \mathcal{L}_{n}^{2} \rightarrow \mathcal{L}_{n}^{2}$ such that $p=\Delta q$ if and only if $\Delta$ is $(I, 0,-I)$-dissipative.

Definition 3.3: A quadratic performance is a quadratic functional $\sigma_{p}(z, d)$ defined as

$$
\sigma_{p}(z, d)=\int_{0}^{\infty}\left[\begin{array}{l}
d(t) \\
z(t)
\end{array}\right]^{T}\left[\begin{array}{cc}
\Pi_{p 1} & \Pi_{p 2} \\
\Pi_{p 2}^{T} & \Pi_{p 3}
\end{array}\right]\left[\begin{array}{l}
d(t) \\
z(t)
\end{array}\right] d t
$$

A system is said to satisfy $\sigma_{p}$-performance criterion over a set of disturbances if the system is well-posed, internally stable and its performance measurement $z$ satisfies $\sigma_{p}(z, d)<0$.

The following theorem gives a sufficient condition for the system that satisfies the performance criterion $\sigma_{p}<0$ over a class of signals $\mathcal{W}$ which can be characterized by IQCs. Based on this theorem, the main stability result of this paper is derived.

Theorem 1: Suppose the operator $\Delta P$ in (6) is $\{X, Y, Z\}$-dissipative, then the interconnected systems (5), (6) satisfies $\sigma_{p}(z, d)$ performance (14), if there exists symmetric matrix $X_{T} \in \mathbf{R}_{S}^{m \times m}, X_{T}>0$, such that the following LMI holds true.

$$
\left[\begin{array}{ccc}
I & 0 & 0 \\
A_{T T} & B_{T S} & B_{T d} \\
\hline 0 & I & 0 \\
A_{S T} & A_{S S} & B_{S d} \\
\hline 0 & 0 & I
\end{array}\right]^{T}\left[\begin{array}{cc|cc|ccc}
0 & X_{T} & 0 & 0 & 0 & 0 \\
X_{T} & 0 & 0 & 0 & 0 & 0 \\
\hline 0 & 0 & X & Y & 0 & 0 \\
0 & 0 & Y^{T} & Z & 0 & 0 \\
\hline 0 & 0 & 0 & 0 & \Pi_{p 1} & \Pi_{p 2} \\
0 & 0 & 0 & 0 & \Pi_{p 2}^{T} & \Pi_{p 3}
\end{array}\right]\left[\begin{array}{cccc}
I & 0 & 0 \\
A_{T T} & B_{T S} & B_{T d} \\
0 & I & 0 \\
C_{T z} & C_{S z} & D_{z d}
\end{array}\right]<0
$$

Proof 1: The proof can be found in [19].

Remark 1: The operator $\Delta P$ used to model the interconnection $v=\Delta P w$ is characterized by several IQCs, $\sigma_{w 1}, \sigma_{w 2}, \ldots, \sigma_{w n}$. In this case the performance can be formulated as a convex feasibility problem over the set of 
IQCs via the lossless S-procedures,

$$
\sigma_{p}(z, d)+\sum_{i=1}^{n} \lambda_{i} \sigma_{w i}(w)<0, \quad \forall w \in \mathcal{L}_{2} .
$$

Remark 2: From the lossless $(D, G)$ scaling theorem for Linear Time Invariant (LTI) systems with Linear Time Varying (LTV) uncertainties, for the contractive operators $(\widetilde{\delta}, \delta$ and $\Delta$ ) listed in lemma 1 , the above stability results are both necessary and sufficient for proper multipliers to model those LTV contractive operators [25]. Geometrically, this sufficient part of proposition 1 can be proved via an separation of graph argument, and the inner matrix in (15) can be interpreted as a hyperplane to separate the graph of the linear time invariant system $G$ and the graph of the time-varying interconnections operators $\Delta$ [20]. The proof of the necessity part follows from the idea proposed in [26] to construct a causal destabilizing operator when strict separation of the two graph is violated. The scalar case $\delta, \widetilde{\delta}$ has been proved in [27],[25] respectively. For the contractive operators listed in lemma 1, the above proposition is a LMI reformulation of the necessary and sufficient condition presented in [25] via an application of the KYP lemma to the LTI system (5).

\section{B. Distributed Stability Conditions}

In this section, we use IQCs to model the interconnection between subsystems. For each of the subsystems $G_{i}, i=i, \ldots, L$, let us consider a specific quadratic form on $\mathbf{R}^{n_{i}} \times \mathbf{R}^{n_{i}}$, such that

$$
\mathcal{P}_{i j}\left(v_{i j}, w_{i j}\right)=\left[\begin{array}{c}
v_{i j} \\
w_{i j}
\end{array}\right]^{T} X_{i j}\left[\begin{array}{c}
v_{i j} \\
w_{i j}
\end{array}\right]
$$

The scaling matrix $X_{i j}$ is further partitioned into four $n_{i j}$ by $n_{i j}$ blocks as

$$
X_{i j}=\left[\begin{array}{cc}
X_{i j}^{11} & X_{i j}^{12} \\
\left(X_{i j}^{12}\right)^{T} & X_{i j}^{22}
\end{array}\right]
$$

The multipliers are chosen to model a set of interconnection operators such that the stability condition for the global system can be equivalently represented as a set of 'decoupled' stability conditions. In this way, it is possible to design distributed controllers for the global system to guarantee global performance.

We are now in a position to state our first analysis conditions regarding the distributed system with interconnections modeled by the a special set of IQCs.

Theorem 2: The interconnected system is well-posed, stable and contractive if there exist symmetric matrices, $X_{T}^{i} \in \mathbf{R}_{S}^{m_{i} \times m_{i}}$ and $X_{i j} \in \mathbf{R}_{S}^{2 n_{i j} \times 2 n_{i j}}, X_{T}^{i}>0$ such that

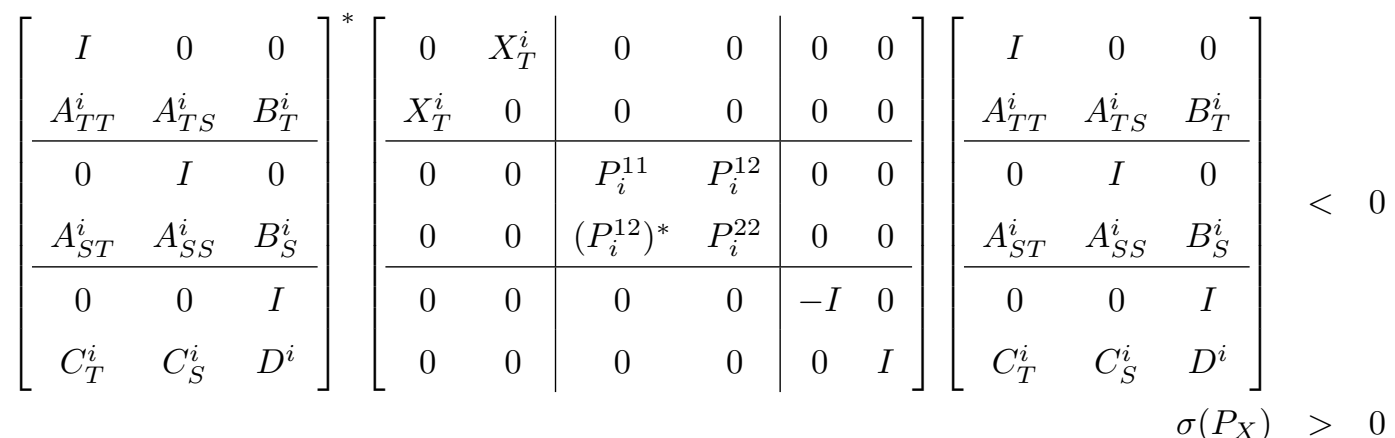


for all $i=1, \ldots, L$. where

$$
\begin{gathered}
P_{i}^{11}=\operatorname{diag}_{1 \leq j \leq L} X_{i j}^{11} \\
P_{i}^{22}=\operatorname{diag}_{1 \leq j \leq L} X_{i j}^{22} \\
P_{i}^{12}=\operatorname{diag}_{1 \leq j \leq L} X_{i j}^{12} \\
\sigma\left(P_{X}\right)=\int_{0}^{\infty}\left[\begin{array}{c}
v \\
w
\end{array}\right]^{T} \mathcal{P}_{X}\left[\begin{array}{c}
v \\
w
\end{array}\right] d t \\
\sum_{1 \leq i, j \leq L} \int_{0}^{\infty}\left[\begin{array}{c}
v_{i j} \\
w_{i j}
\end{array}\right]^{T}\left[\begin{array}{cc}
X_{i j}^{11} & X_{i j}^{12} \\
\left(X_{i j}^{12}\right)^{T} & X_{i j}^{22}
\end{array}\right]\left[\begin{array}{c}
v_{i j} \\
w_{i j}
\end{array}\right] d t
\end{gathered}
$$

Proof 2: The above theorem is a reformulation of the global performance condition from theorem 1 by utilizing the structural information of the IQCs and diagonal structure of the global system.

We now consider different IQCs that can be modeled by $\sigma\left(P_{X}\right)$ in Theorem 2 by choosing different scaling matrices $X_{i j}$ to model the interconnection operator $\Delta_{i j}$.

1) Perfect Interconnections: Here we assume that $\Delta_{i, j}=I_{n_{i j}}, \forall i, j$, that is, at any instance $t$

$$
v_{i j}(t)=w_{j i}(t), \quad \forall i, j, t \geq 0
$$

In this case,

$$
\begin{aligned}
\sigma\left(P_{X}\right) & =\sum_{1 \leq i, j \leq L} \int_{0}^{\infty}\left[\begin{array}{c}
v_{i j} \\
w_{i j}
\end{array}\right]^{T} X_{i j}\left[\begin{array}{c}
v_{i j} \\
w_{i j}
\end{array}\right] d t \\
& =\sum_{i, j, i \geq j} \int_{0}^{\infty}\left[\begin{array}{c}
v_{i j} \\
w_{i j}
\end{array}\right]^{T}\left[\begin{array}{cc}
X_{i j}^{11}+X_{j i}^{22} & X_{i j}^{12}+\left(X_{j i}^{12}\right)^{T} \\
\left(X_{i j}^{12}\right)^{T}+X_{j i}^{12} & X_{i j}^{22}+X_{j i}^{11}
\end{array}\right]\left[\begin{array}{c}
v_{i j} \\
w_{i j}
\end{array}\right]
\end{aligned}
$$

Suppose we choose for all $1 \leq i, j \leq L$

$$
\begin{aligned}
X_{i j}^{11}+X_{j i}^{22} & =0 \\
X_{i j}^{12}+\left(X_{j i}^{12}\right)^{T} & =0
\end{aligned}
$$

With the above parameterization, $\sigma\left(P_{X_{\text {ideal }}}\right)=0$ and the family of multipliers $X_{\text {ideal }}$ can be characterized by the following two sets

$$
\left\{X_{i j}^{11} \in \mathbf{R}_{S}^{n_{i j} \times n_{i j}}, i, j=1, \ldots, L\right\}
$$

and

$$
\left\{X_{i j}^{12} \in \mathbf{R}_{S}^{n_{i j} \times n_{i j}}: X_{i i}^{12} \text { skew-symmetric, } 1 \leq j \leq i \leq L\right\}
$$


Proposition 1: The interconnected system (5), (6) is well-posed, stable and contractive for all $\Delta_{i j}=I_{n_{i j}}$ if there exist symmetric matrices, $X_{T}^{i} \in \mathbf{R}_{S}^{m_{i} \times m_{i}}$ and $X_{i j} \in \mathbf{R}_{S}^{2 n_{i j} \times 2 n_{i j}}, X_{T}^{i}>0$ such that

$$
\left[\begin{array}{ccc}
I & 0 & 0 \\
A_{T T}^{i} & A_{T S}^{i} & B_{T}^{i} \\
\hline 0 & I & 0 \\
A_{S T}^{i} & A_{S S}^{i} & B_{S}^{i} \\
\hline 0 & 0 & I \\
C_{T}^{i} & C_{S}^{i} & D^{i}
\end{array}\right]^{*}\left[\begin{array}{cc|cc|cc}
0 & X_{T}^{i} & 0 & 0 & 0 & 0 \\
X_{T}^{i} & 0 & 0 & 0 & 0 & 0 \\
\hline 0 & 0 & P_{i}^{11} & P_{i}^{12} & 0 & 0 \\
0 & 0 & \left(P_{i}^{12}\right)^{*} & P_{i}^{22} & 0 & 0 \\
\hline 0 & 0 & 0 & 0 & -I & 0 \\
0 & 0 & 0 & 0 & 0 & I
\end{array}\right]\left[\begin{array}{cccc}
I & 0 & 0 \\
A_{T T}^{i} & A_{T S}^{i} & B_{T}^{i} \\
0 & I & 0 \\
A_{S T}^{i} & A_{S S}^{i} & B_{S}^{i} \\
0 & 0 & I \\
C_{T}^{i} & C_{S}^{i} & D^{i}
\end{array}\right]<0
$$

for all $i=1, \ldots, L$. where

$$
\begin{aligned}
P_{i}^{11} & =\operatorname{diag}_{1 \leq j \leq L} X_{i j}^{11} \\
P_{i}^{22} & =\operatorname{diag}_{1 \leq j \leq L}-X_{j i}^{11} \\
P_{i}^{12} & =\operatorname{diag}\left(\operatorname{diag}_{1 \leq j \leq i} X_{i j}^{12}, \operatorname{diag}_{i \leq j \leq i}-\left(X_{j i}^{12}\right)^{T}\right)
\end{aligned}
$$

In [1], the signal space considered for the perfect interconnection case is the vector space $R^{N}$ instead of $\mathcal{L}_{2}^{N}$. Note that when viewing the $v_{i j}(t)=w_{j i}(t)$ as algebraic constraints in the vector space $R^{N}$, the well-posedness of the above proposition need to be reevaluated (in $R^{N}$ ). Based on the matrix form $S$-procedure developed in [24], [22], the well-posedness condition in $R^{N}$ is also guaranteed by (30).

2) Directed Interconnection with $\Delta_{i j}=\delta_{i j} I_{n_{i j}}$ : Let us now consider a new class of interconnected systems with $\Delta_{i j}=\delta_{i j} I_{n_{i j}}$ and $\left\|\delta_{i j}\right\| \leq 1$.

We are seeking a new IQC to model such interconnections. Suppose we parameterize the multipliers $X_{i j}$ by the following sets of matrices

$$
\left\{X_{i j}^{11} \in \mathbf{R}_{S}^{n_{i j} \times n_{i j}}, X_{i j}^{11}<0, i, j=1, \ldots, L\right\}
$$

and

$$
\left\{X_{i j}^{22}=X_{j i}^{11}, X_{i j}^{12}=0\right\}
$$

In this case, it is straightforward to verify

$$
\begin{aligned}
& \sigma\left(P_{X_{\delta}}\right)=\frac{1}{2} \sum_{1 \leq i, j \leq L}\left\langle\left[\begin{array}{c}
v_{i j} \\
w_{i j}
\end{array}\right],\left[\begin{array}{cc}
X_{i j}^{11} & 0 \\
0 & -X_{j i}^{11}
\end{array}\right]\left[\begin{array}{c}
v_{i j} \\
w_{i j}
\end{array}\right]\right\rangle+\left\langle\left[\begin{array}{c}
v_{j i} \\
w_{j i}
\end{array}\right],\left[\begin{array}{cc}
X_{j i}^{11} & 0 \\
0 & -X_{i j}^{11}
\end{array}\right]\left[\begin{array}{c}
v_{j i} \\
w_{j i}
\end{array}\right]\right\rangle \\
& =\sum_{1 \leq j \leq i \leq L}<v_{i j}, X_{i j}^{11} v_{i j}>-<w_{j i}, X_{i j}^{11} w_{j i}> \\
& \geq 0
\end{aligned}
$$

Following similar arguments, we have the following propositions(2, 4). The sufficient part can be similarly proved as in Proposition 1, and the necessity part follows from the lossless- $(D, G)$-scaling theorem for LTV uncertainties. The details of the proof are omitted because of space considerations, they can be viewed as reformulations of the $(D, G)$-scaling theorem for the distributed systems. 
Proposition 2: The interconnected system (5), (6) is well-posed, stable and contractive for all $\Delta_{i j}=I_{n_{i j}} \delta,\|\delta\| \leq$ 1 if and only if there exist symmetric matrices, $X_{T}^{i} \in \mathbf{R}_{S}^{m_{i} \times m_{i}}, X_{T}^{i}>0$ and for all $i, j=1, \ldots, L, X_{i j} \in$ $\mathbf{R}_{S}^{n_{i j} \times n_{i j}}, X_{i j}^{11}<0$ and LMI (30) are satisfied for all $i=1 \ldots, L$, with $P_{i}^{11}=\operatorname{diag}_{j}\left(X_{i j}^{11}\right), P_{i}^{22}=\operatorname{diag}_{j}\left(-X_{j i}\right)^{11}$ and $P_{i}^{12}=0$.

Proposition 3: The interconnected system (5), (6) is well-posed, stable and contractive for all LTV $\Delta_{i j}=$ $I_{n_{i j}} \widetilde{\delta},\|\widetilde{\delta}\| \leq 1, \widetilde{\delta}$ self-adjoint if and only if there exist symmetric matrices, $X_{T}^{i} \in \mathbf{R}_{S}^{m_{i} \times m_{i}}, X_{T}^{i}>0$ and for all $i, j=1, \ldots, L, X_{i j}^{11}, X_{i j}^{12} \in \mathbf{R}_{S}^{n_{i j} \times n_{i j}}, X_{i j}^{11}<0$ and LMI (30) are satisfied for all $i=1 \ldots, L$, with $P_{i}^{11}, P_{i}^{22}$ and $P_{i}^{12}$ defined as (31), (32), (33).

Proposition 4: The interconnected system (5), (6) is well-posed, stable and contractive for all LTV $\Delta_{i j},\left\|\Delta_{i j}\right\| \leq$ 1 , if and only if there exist symmetric matrices, $X_{T}^{i} \in \mathbf{R}_{S}^{m_{i} \times m_{i}}, X_{T}^{i}>0$ and for all $i, j=1, \ldots, L, d_{i j}<0$, $X_{i j}^{11}=d_{i j}^{11} I_{n_{i j}}$ and LMI (30) are satisfied for all $i=1 \ldots, L$, with $P_{i}^{11}=\operatorname{diag}_{j}\left(X_{i j}^{11}\right), P_{i}^{22}=\operatorname{diag}_{j}\left(-X_{j i}\right)^{11}$ and $P_{i}^{12}=0$.

Before we apply the stability analysis results for synthesis, the following commends are given. Theorem 2 unifies the stability results for different interconnections which can be modeled as integral quadratic constraints. This theorem renders the performance specification based on the interconnected uncertain systems to an explicit expression through the S-procedure, where the multipliers $X_{i j}$ are shaped by the structure and properties of the interconnection operator $\Delta_{i j}$. Theorem 2 reflects the simple idea of topological separation of the graph generated via the LTI plant and the LTV uncertainty.

\section{Distributed Controller Synthesis Conditions}

The synthesis part of this paper follows the same lines of derivation presented in [1], which are based on the extended elimination lemma. We want to point out that for the synthesis condition corresponding to Theorem 2 in [1], $n_{i j}^{K}=n_{i j}$ is enough since the inertia constraints for the closed-loop system are satisfied if the conditions in (49), (50) are feasible and the multipliers are nonsingular.

Now let us consider each of subsystems $G_{i}$ with control input $u_{i}$ and a measured output $y_{i}$, in addition to the signals given in (1), such that

$$
\begin{aligned}
{\left[\begin{array}{c}
\dot{x}_{i}(t) \\
w_{i}(t) \\
z_{i}(t) \\
y_{i}(t)
\end{array}\right] } & =\left[\begin{array}{cccc}
A_{T T}^{i} & A_{T S}^{i} & B_{T d}^{i} & B_{T u}^{i} \\
A_{S T}^{i} & A_{S S}^{i} & B_{S}^{i} & B_{S u}^{i} \\
C_{T}^{i} & C_{S}^{i} & D^{i} & D_{z u}^{i} \\
C_{T y}^{i} & C_{S y}^{i} & D_{y d}^{i} & D_{y u}^{i}
\end{array}\right]\left[\begin{array}{c}
x_{i}(t) \\
v_{i}(t) \\
d_{i}(t) \\
u_{i}(t)
\end{array}\right] \\
v_{i j} & =\Delta_{j i} w_{j i}
\end{aligned}
$$

for all $t \geq 0$ and $i=1, \ldots, L . \Delta_{j i}$ is an operator used here to specify the interconnection. In the rest of this paper, without loss of generality, we assume that $D_{y u}^{i}=0, \forall i$. Similarly to the controller considered in the LPV literature, we are seeking controllers with 'similar' structure as the plant: another interconnected system $K$ with subsystems 
$K_{i}, i=1, \ldots, L$ given by

$$
\left[\begin{array}{c}
\dot{x}_{i}^{K}(t) \\
w_{i}^{K}(t) \\
u_{i}(t)
\end{array}\right]\left[\begin{array}{ccc}
\left(A_{T T}^{i}\right)_{K} & \left(A_{T S}^{i}\right)_{K} & \left(B_{T}^{i}\right)_{K} \\
\left(A_{S T}^{i}\right)_{K} & \left(A_{S S}^{i}\right)_{K} & \left(B_{S}^{i}\right)_{K} \\
\left(C_{T}^{i}\right)_{K} & \left(C_{S}^{i}\right)_{K} & D_{K}^{i}
\end{array}\right]\left[\begin{array}{c}
x_{i}^{K}(t) \\
v_{i}^{K}(t) \\
y_{i}(t)
\end{array}\right]
$$

such that the closed loop system is well-posed, stable and contractive. In addition, we require $n_{i j}^{K}=0$ whenever $n_{i j}=0$, which means that if there is no communication between $G_{i}$ and $G_{j}$, there is no communication between controllers $K_{i}$ and $K_{j}$, either.

The state vector for the subsystem $x_{c}^{i}$ has size $m_{i}+m_{i}^{K}$,

$$
x_{c}^{i}=\left[\begin{array}{c}
x_{i} \\
x_{i}^{K}
\end{array}\right] .
$$

The interconnection signal $w_{i j}^{C}, v_{i j}^{C}$ has size $n_{i j}^{C}=n_{i j}+n_{i j}^{K}$,

$$
\begin{aligned}
& w_{i j}^{C}=\left[\begin{array}{l}
w_{i j} \\
w_{i j}^{K}
\end{array}\right] \\
& v_{i j}^{C}=\left[\begin{array}{c}
v_{i j} \\
v_{i j}^{K}
\end{array}\right]
\end{aligned}
$$

Besides, since the controller $K$ and the plant $G$ share the same communication channel between each subsystem, we further require

$$
v_{i j}^{C}=\Delta_{j i} w_{j i}^{C}
$$

Proposition 5: There exist distributed controllers with state representation (36) with $n_{i j}^{K}=n_{i j}$ and interconnection $\Delta_{i j}=I$ such that the closed-loop system is well-posed, stable and contractive if and only if for all $i=1, \ldots, L$, there exist symmetric matrices $\left(X_{T}^{i}\right)_{G},\left(Y_{T}^{i}\right)_{G} \in \mathbf{R}_{S}^{m_{i} \times m_{i}}\left(X_{i j}\right)_{G}^{T},\left(Y_{i j}\right)_{G}^{T} \in \mathbf{R}_{S}^{n_{i j} \times n_{i j}}$ for all $i, j=1, \ldots, L$, and $\left(X_{i j}^{11}\right)_{G},\left(Y_{i j}^{11}\right)_{G} \in \mathbf{R}_{S}^{n_{i j} \times n_{i j}}$ for all $i, j=1, \ldots, L$ and $\left(X_{i j}^{12}\right)_{G},\left(Y_{i j}^{12}\right)_{G} \in \mathbf{R}^{n_{i j} \times n_{i j}}$ for $i \geq j$, with $\left(X_{i i}^{12}\right)_{G},\left(Y_{i i}^{12}\right)_{G}$ skew-symmetric such that $\left(X_{T}^{i}\right)>0,\left(Y_{T}^{i}\right)>0$ and LMIs (49),(49),(49) are satisfied, and $\Psi^{i}, \Phi^{i}$ are defined as (40), (41) respectively.

$$
\begin{aligned}
\Psi^{i} & =\operatorname{ker}\left[\begin{array}{lll}
C_{T y}^{i} & C_{S y}^{i} & D_{y d}^{i}
\end{array}\right] \\
\Phi^{i} & =\operatorname{ker}\left[\begin{array}{lll}
\left(B_{T u}^{i}\right)^{T} & \left(B_{S u}^{i}\right)^{T} & \left(D_{z u}^{i}\right)^{T}
\end{array}\right]
\end{aligned}
$$


and

$$
\begin{aligned}
& \left(Z_{i}^{11}\right)=\operatorname{diag}_{1 \leq j \leq L}\left(X_{i j}^{11}\right)_{G} \\
& \left(Z_{i}^{22}\right)=-\operatorname{diag}_{1 \leq j \leq L}\left(X_{j i}^{11}\right)_{G} \\
& \left.\left.\left(Z_{i}^{22}\right)=\operatorname{diag}\left\{\operatorname{diag}_{1 \leq j \leq i} X_{i j}^{12}\right)_{G},-\operatorname{diag}_{i<j \leq L} X_{j i}^{12}\right)_{G}^{*}\right\} \\
& \left(\widetilde{Z}_{i}^{11}\right)=\operatorname{diag}_{1 \leq j \leq L}\left(Y_{i j}^{11}\right)_{G} \\
& \left(\widetilde{Z}_{i}^{22}\right)=-\operatorname{diag}_{1 \leq j \leq L}\left(Y_{j i}^{11}\right)_{G} \\
& \left.\left.\left(\widetilde{Z}_{i}^{22}\right)=\operatorname{diag}\left\{\operatorname{diag}_{1 \leq j \leq i} Y_{i j}^{12}\right)_{G},-\operatorname{diag}_{i<j \leq L} Y_{j i}^{12}\right)_{G}^{I}\right\} \\
& \left(\Psi^{i}\right)^{*}\left[\begin{array}{ccc}
I & 0 & 0 \\
A_{T T}^{i} & A_{T S}^{i} & B_{T}^{i} \\
\hline 0 & I & 0 \\
A_{S T}^{i} & A_{S S}^{i} & B_{S}^{i} \\
\hline 0 & 0 & I \\
C_{T}^{i} & C_{S}^{i} & D^{i}
\end{array}\right]^{*}\left[\begin{array}{cc|cc|cc}
0 & \left(X_{T}^{i}\right)_{G} & 0 & 0 & 0 & 0 \\
\left(X_{T}^{i}\right)_{G} & 0 & 0 & 0 & 0 & 0 \\
\hline 0 & 0 & \left(Z_{i}^{11}\right)_{G} & \left(Z_{i}^{12}\right)_{G} & 0 & 0 \\
0 & 0 & \left(Z_{i}^{12}\right)_{G}^{*} & \left(Z_{i}^{22}\right)_{G} & 0 & 0 \\
\hline 0 & 0 & 0 & 0 & I & 0 \\
0 & 0 & 0 & 0 & 0 & -I
\end{array}\right]\left[\begin{array}{ccc}
I & 0 \\
A_{T T}^{i} & A_{T S}^{i} & B_{T}^{i} \\
\hline 0 & 0 \\
A_{S T}^{i} & A_{S S}^{i} & B_{S}^{i} \\
\hline 0 & 0 & I \\
C_{T}^{i} & C_{S}^{i} & D^{i}
\end{array}\right] \Psi^{i}<0
\end{aligned}
$$

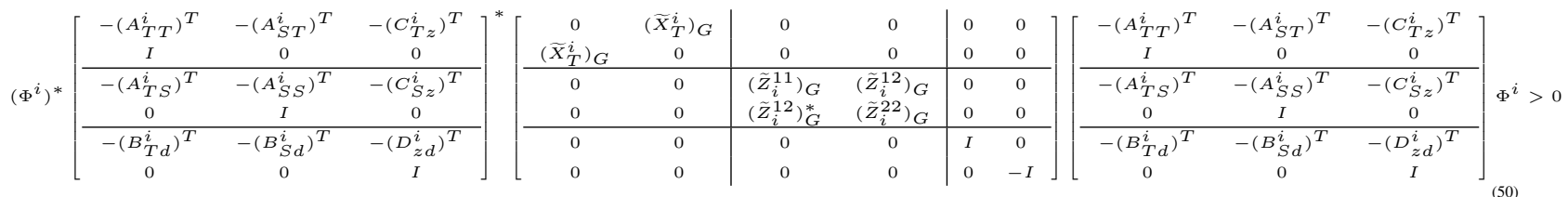

$$
\begin{aligned}
& {\left[\begin{array}{cc}
\left(X_{T}^{i}\right)_{G} & I \\
I & \left(Y_{T}^{i}\right)_{G}
\end{array}\right]>0}
\end{aligned}
$$

Proof 3: Notice that, the closed loop system for the individual subsystem with the controller described by (36) is linear in the controller's parameter $\Theta_{i}$ with

$$
\Theta_{i}=\left[\begin{array}{ccc}
\left(A_{T T}^{i}\right)_{K} & \left(A_{T S}^{i}\right)_{K} & \left(B_{T}^{i}\right)_{K} \\
\left(A_{S T}^{i}\right)_{K} & \left(A_{S S}^{i}\right)_{K} & \left(B_{S}^{i}\right)_{K} \\
\left(C_{T}^{i}\right)_{K} & \left(C_{S}^{i}\right)_{K} & D_{K}^{i}
\end{array}\right]
$$

If the elimination lemma from [23](see appendix) is applied to each individual stability condition derived in Proposition 1 (30) for the closed-loop system, the necessity part follows instantly. The sufficient part follows using similar techniques to construct the extended multiplier for the overall interconnection $w_{i j}^{C}, v_{j i}^{C}$ [24].

(Necessity) Suppose there exist symmetric matrices $\left(X_{T}^{i}\right)^{C} \in R_{S}^{m_{i}^{C} \times m_{i}^{C}}$ and $X_{i j}^{11} \in R_{S}^{n_{i j}^{C} \times n_{i j}^{C}}$ for all $i, j=$ $1, \ldots, L$, and $\left(X_{i j}^{12}\right)_{C} \in R^{n_{i j}^{C} \times n_{i j}^{C}}$ for all $i \geq j$, with $\left(X_{i i}^{12}\right)_{C}$ skew symmetric, such that $\left(X_{T}^{i}\right)_{C}>0$ and (30) is satisfied, then try to show that (49), (50) are satisfied. Let

$$
P_{C}^{i}=\left[\begin{array}{cc|cc}
0 & \left(X_{T}^{i}\right)_{C} & 0 & 0 \\
\left(X_{T}^{i}\right)_{C} & 0 & 0 & 0 \\
\hline 0 & 0 & \left(Z_{i}^{11}\right)_{C} & \left(Z_{i}^{12}\right)_{C} \\
0 & 0 & \left(Z_{i}^{12}\right)_{C}^{*} & \left(Z_{i}^{22}\right)_{C}
\end{array}\right]
$$


and

$$
\begin{aligned}
\widetilde{P}_{C}^{i} & =\left(P_{C}^{i}\right)^{-1} \\
& =\left[\begin{array}{cc|cc}
0 & \left(\widetilde{X}_{T}^{i}\right)_{C} & 0 & 0 \\
\left(\widetilde{X}_{T}^{i}\right)_{C} & 0 & 0 & 0 \\
\hline 0 & 0 & \left(\widetilde{Z}_{i}^{11}\right)_{C} & \left(\widetilde{Z}_{i}^{12}\right)_{C} \\
0 & 0 & \left(\widetilde{Z}_{i}^{12}\right)_{C}^{*} & \left(\widetilde{Z}_{i}^{22}\right)_{C}
\end{array}\right]
\end{aligned}
$$

Suppose $P_{C}^{i}$ is nonsingular. Applying the elimination lemma 2 to the stability condition (30) of the closed-loop system, we have

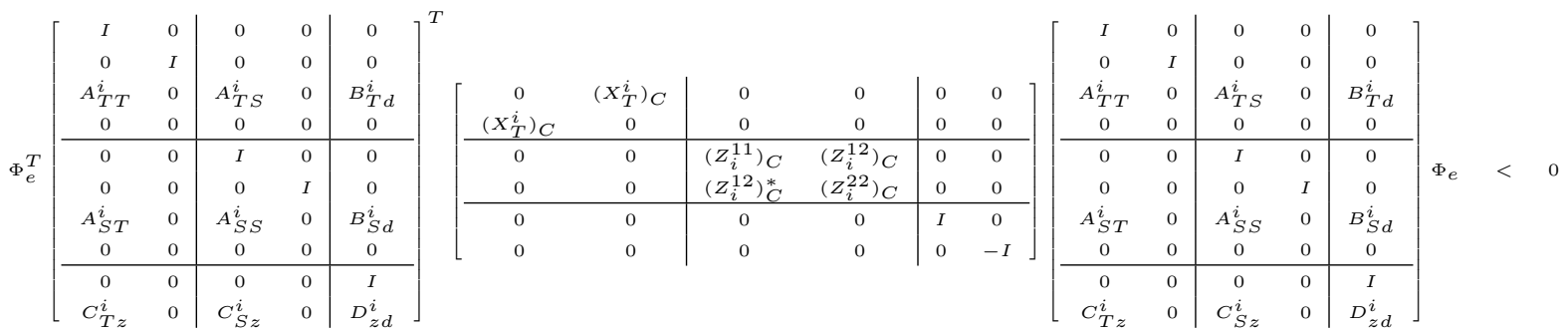

Due to the zero block of $\Phi_{e}$, it is clear that this is the same as

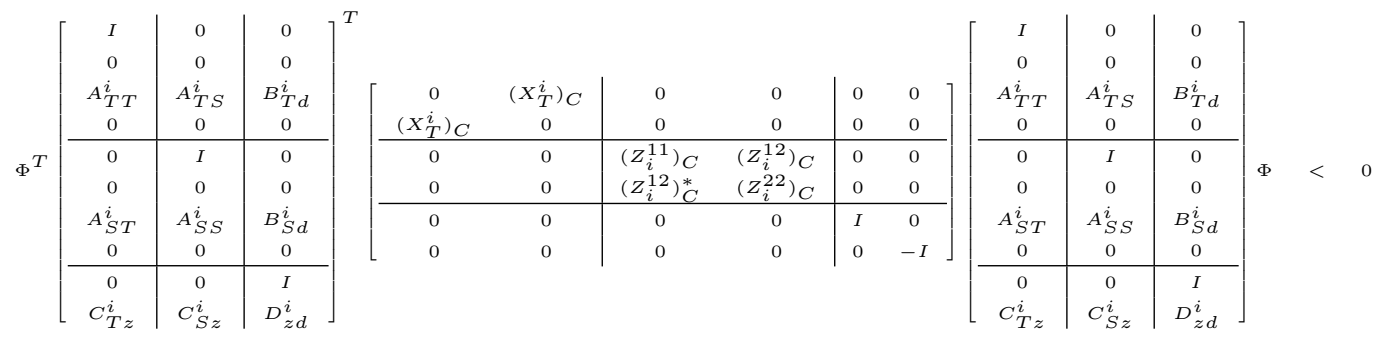

The four zero block rows in the outer factors allow us to simplify this inequality to obtain (49). Inequality (50) can be derived via similar argument with respect to $\widetilde{P}_{C}^{i}$.

(Sufficiency) If the LMIs (49), (49), (51) are satisfied, we can find the block multipliers $\left(X_{T}^{i}\right)_{C},\left(Z_{i}\right)_{C}$, such that proposition (30) is satisfied. For this, it is enough to show that

$$
\left[\begin{array}{cc}
\left(X_{T}^{i}\right)_{G} & \left(X_{T}^{i}\right)_{G K} \\
\left(\left(X_{T}^{i}\right)_{G K}\right)^{*} & \left(X_{T}^{i}\right)_{K}
\end{array}\right]^{-1}=\left[\begin{array}{cc}
\left(Y_{T}^{i}\right)_{G} & \left(Y_{T}^{i}\right)_{G K} \\
\left(\left(Y_{T}^{i}\right)_{G K}\right)^{*} & \left(Y_{T}^{i}\right)_{K}
\end{array}\right]>0
$$

This result can be proved via lemma 3 .

$$
\begin{aligned}
\left(X_{i j}^{-1}\right)_{C} & =\left[\begin{array}{ll}
\left(X_{i j}^{11}\right)_{C} & \left(X_{i j}^{12}\right)_{C} \\
\left(X_{i j}^{12}\right)_{C}^{*} & -\left(X_{j i}^{11}\right)_{C}
\end{array}\right] \\
& =\left[\begin{array}{cc}
\left(Y_{i j}^{11}\right)_{C} & \left(Y_{i j}^{12}\right)_{C} \\
\left(Y_{i j}^{12}\right)_{C}^{*} & -\left(Y_{j i}^{11}\right)_{C}
\end{array}\right]
\end{aligned}
$$

for all $i \geq j$. Notice that for $i=j,\left(X_{i j}\right)_{C} \in \kappa_{C}^{2 n_{i j}^{C} \times 2 n_{i j}^{C}}$, which can be proved via lemma 5 , and for the general case $i>j$, this can be proved via lemma 4 . 
In correspondence to Proposition 2, Proposition 3, Proposition 4, the following synthesis condition can be proved similarly to Proposition 5.

Proposition 6: There exist distributed controllers with state representation (36) with $n_{i j}^{K}=n_{i j}$ and interconnection $\Delta_{i j}=\delta I,\|\delta\| \leq 1$ such that the closed-loop system is well-posed, stable and contractive if and only if for all $i=1, \ldots, L$, there exist symmetric matrices $\left(X_{T}^{i}\right)_{G},\left(Y_{T}^{i}\right)_{G} \in \mathbf{R}_{S}^{m_{i} \times m_{i}}\left(X_{i j}\right)_{G}^{T},\left(Y_{i j}\right)_{G}^{T} \in \mathbf{R}_{S}^{n_{i j} \times n_{i j}}$ for all $i, j=1, \ldots, L$, and $\left(X_{i j}^{11}\right)_{G},\left(Y_{i j}^{11}\right)_{G} \in \mathbf{R}_{S}^{n_{i j} \times n_{i j}},\left(X_{i j}^{11}\right)_{G}<0,\left(Y_{i j}^{11}\right)_{G}<0$ for all $i, j=1, \ldots, L$ and $\left(X_{i j}^{12}\right)_{G},\left(Y_{i j}^{12}\right)_{G} \in \mathbf{R}^{n_{i j} \times n_{i j}}$ for $i, j=1 \ldots, L$, with $\left(X_{i j}^{12}\right)_{G}=\left(Y_{i j}^{12}\right)_{G}=0$ such that $\left(X_{T}^{i}\right)>0,\left(Y_{T}^{i}\right)>0$ and LMIs (49),(49),(49) are satisfied, and $\Psi^{i}, \Phi^{i}$ are defined as (40), (41) respectively.

Proposition 7: There exist distributed controllers with state representation (36) with $n_{i j}^{K}=n_{i j}$ and interconnection $\Delta_{i j}=\widetilde{\delta} I$ such that the closed-loop system is well-posed, stable and contractive if and only if for all $i=1, \ldots, L$, there exist symmetric matrices $\left(X_{T}^{i}\right)_{G},\left(Y_{T}^{i}\right)_{G} \in \mathbf{R}_{S}^{m_{i} \times m_{i}}\left(X_{i j}\right)_{G}^{T},\left(Y_{i j}\right)_{G}^{T} \in \mathbf{R}_{S}^{n_{i j} \times n_{i j}}$ for all $i, j=1, \ldots, L$, and $\left(X_{i j}^{11}\right)_{G},\left(Y_{i j}^{11}\right)_{G} \in \mathbf{R}_{S}^{n_{i j} \times n_{i j}},\left(X_{i j}^{11}\right)_{G}<0,\left(Y_{i j}^{11}\right)_{G}<0$ for all $i, j=1, \ldots, L$ and $\left(X_{i j}^{12}\right)_{G},\left(Y_{i j}^{12}\right)_{G} \in \mathbf{R}^{n_{i j} \times n_{i j}}$ for $i \geq j$, with $\left(X_{i j}^{12}\right)_{G},\left(Y_{i j}^{12}\right)_{G}$ skew-symmetric such that $\left(X_{T}^{i}\right)_{G}>0,\left(Y_{T}^{i}\right)_{G}>0$ and LMIs (49),(49),(49) are satisfied, and $\Psi^{i}, \Phi^{i}$ are defined as (40), (41) respectively.

Proposition 8: There exist distributed controllers with state representation (36) with $n_{i j}^{K}=n_{i j}$ and interconnection $\Delta_{i j},\left\|\Delta_{i j}\right\| \leq 1$ such that the closed-loop system is well-posed, stable and contractive if and only if for all $i=1, \ldots, L$, there exist symmetric matrices $\left(X_{T}^{i}\right)_{G},\left(Y_{T}^{i}\right)_{G} \in \mathbf{R}_{S}^{m_{i} \times m_{i}}$, and $x_{i j}, y_{i j} \in \mathbf{R}$ such that $x_{i j}<0, y_{i j}<0,\left(X_{i j}^{11}\right)_{G}=x_{i j} I_{n_{i j}},\left(Y_{i j}^{11}\right)_{G}=y_{i j} I_{n_{i j}}$ for all $i, j=1, \ldots, L$ and $\left(X_{i j}^{12}\right)_{G},\left(Y_{i j}^{12}\right)_{G} \in$ $\mathbf{R}^{n_{i j} \times n_{i j}},\left(X_{i j}^{12}\right)_{G}=\left(Y_{i j}^{12}\right)_{G}=0$ for $i \geq j$ such that such that $\left(X_{T}^{i}\right)_{G}>0,\left(Y_{T}^{i}\right)_{G}>0$ and LMIs (49),(49),(49) are satisfied, and $\Psi^{i}, \Phi^{i}$ are defined as (40), (41) respectively.

\section{CONCLUSION}

In this paper, we derived stability conditions for distributed systems with IQC constraints that model the internal interconnections. The sufficient stability results follow from an application of the S-procedure and can be proved via a graph separation argument. Our stability theorem expresses the condition to guarantee global performance with implicit uncertainty interconnections as one explicit conditions with design multipliers parameterized by the uncertainty. Controller synthesis techniques from the gain-scheduling literature is also introduced in this paper for distributed controllers design. However, questions regarding the performance limitations for these distributed controllers compared to centralized controllers remain open research topics.

\section{APPENDIX}

The following result is basically an extension of the well-known elimination lemma to a quadratic matrix inequality. It is convenient for elimination of controller parameters from the synthesis conditions.

Lemma 2: Elimination Lemma [23]. Let $P$ be a symmetric matrix with inertia in $(P)=(m, 0, n)$ and $C \in \mathbf{R}^{n \times m}$. 
The quadratic matrix inequality,

$$
\left[\begin{array}{c}
I \\
A^{T} X B+C
\end{array}\right]^{T} P\left[\begin{array}{c}
I \\
A^{T} X B+C
\end{array}\right]<0
$$

in the unstructured unknown matrix variable $X$ has a solution if and only if

$$
\begin{array}{r}
B_{\perp}^{T}\left[\begin{array}{c}
I \\
C
\end{array}\right]^{T} P\left[\begin{array}{c}
I \\
C
\end{array}\right] B_{\perp}<0 \\
A_{\perp}^{T}\left[\begin{array}{c}
-C^{T} \\
I
\end{array}\right]^{T} P^{-1}\left[\begin{array}{c}
-C^{T} \\
I
\end{array}\right] A_{\perp}>0
\end{array}
$$

Lemma 3: [18] Suppose $S_{1}, R_{1} \in \mathbf{R}_{S}^{n \times n}$, with $S_{1}>0, R_{1}>0$. Let $m$ be a positive integer. Then there exists matrices $S_{2} \in \mathbf{R}^{n \times m}, S_{3} \in \mathbf{R}_{S}^{m \times m}$, and

$$
\left[\begin{array}{cc}
S_{1} & S_{2} \\
S_{2}^{T} & S_{3}
\end{array}\right]>0 \text { and }\left[\begin{array}{cc}
S_{1} & S_{2} \\
S_{2}^{T} & S_{3}
\end{array}\right]^{-1}=\left[\begin{array}{cc}
R_{1} & R_{2} \\
R_{2}^{T} & R_{3}
\end{array}\right]
$$

if and only if

$$
\left[\begin{array}{ll}
S_{1} & I_{n} \\
I_{n} & R_{1}
\end{array}\right] \geq 0 \text { and rank }\left[\begin{array}{cc}
S_{1} & I_{n} \\
I_{n} & R_{1}
\end{array}\right] \leq n+m
$$

Let us define the following four types of matrices $\chi_{G}^{2 n \times 2 n}, \kappa_{G}^{2 n \times 2 n}, \chi_{C}^{2(n+m) \times 2(n+m)}, \kappa_{C}^{2(n+m) \times 2(n+m)}$

$$
\begin{aligned}
\chi_{G}^{2 n \times 2 n} & =\left\{\Theta: \Theta=\left[\begin{array}{ll}
\Theta_{11} & \Theta_{12} \\
\Theta_{12}^{*} & \Theta_{22}
\end{array}\right], \Theta_{11}, \Theta_{22} \in \mathbf{R}_{S}^{n \times n}, \Theta_{12} \in \mathbf{R}^{n \times n}\right\} \\
\kappa_{G}^{2 n \times 2 n} & =\left\{\Theta: \Theta=\left[\begin{array}{cc}
\Theta_{11} & \Theta_{12} \\
\Theta_{12}^{*} & -\Theta_{11}
\end{array}\right], \Theta_{11} \in \mathbf{R}_{S}^{n \times n}, \Theta_{12} \in \mathbf{R}^{n \times n}, \Theta_{12}^{*}=-\Theta_{12}\right\} \\
\chi_{C}^{2(n+m) \times 2(n+m)} & =\left\{\Theta: \Theta=\left[\begin{array}{cc}
\Theta_{11} & \Theta_{12} \\
\Theta_{12}^{*} & \Theta_{22}
\end{array}\right], \Theta_{11}, \in \chi_{G}^{2 n \times 2 n}, \Theta_{22} \in \chi_{G}^{2 m \times 2 m}, \Theta_{12} \in \mathbf{R}^{2 n \times 2 m}\right\} \\
\kappa_{C}^{2(n+m) \times 2(n+m)} & =\left\{\Theta: \Theta=\left[\begin{array}{cc}
\Theta_{11} & \Theta_{12} \\
\Theta_{12}^{*} & \Theta_{22}
\end{array}\right], \Theta_{11} \in \kappa_{G}^{2 n \times 2 n}, \Theta_{22} \in \kappa_{G}^{2 m \times 2 m}, \Theta_{12} \in \mathbf{R}^{2 n \times 2 m}, \Theta_{12}^{*}=-\Theta(606)\right.
\end{aligned}
$$

Lemma 4: Suppose $S_{1}, R_{1} \in \chi_{G}^{2 n \times 2 n}$, then for some $m$, there exsit a matrix $S, R \in \chi_{C}^{2(n+m) \times 2(n+m)}$, suth that,

$$
S=\left[\begin{array}{cc}
S_{1} & S_{2} \\
S_{2}^{*} & S_{3}
\end{array}\right]^{-1}=\left[\begin{array}{ll}
R_{1} & R_{2} \\
R_{2}^{*} & R_{3}
\end{array}\right]=R
$$

Proof 4: Suppose $N=\left(S_{1}-R_{1}^{-1}\right)^{-1}$ is nonsingular, for any $m \geq n$, choose $S_{3} \in \mathbf{R}_{S}$ such that, $\mathrm{in}_{+}\left(S_{3}\right) \geq$ in $_{-}(N)$, in ${ }_{-}\left(S_{3}\right) \geq$ in $_{-}(N)$, then there exist $T$, such that

$$
N^{-1}=T S_{3}^{-1} T^{*}
$$

choose $S_{3}=T$, then (4) are satisfied with $S_{3}, S_{2}$ specified above and $R_{2}, R_{3}$ can be easily determined. 
Lemma 5: Suppose $S_{1}, R_{1} \in \kappa_{G}^{2 n \times 2 n}$, then for some $m$, there exsit a matrix $S, R \in \kappa_{C}^{2(n+m) \times 2(n+m)}$, suth that,

$$
S=\left[\begin{array}{ll}
S_{1} & S_{2} \\
S_{2}^{*} & S_{3}
\end{array}\right]^{-1}=\left[\begin{array}{cc}
R_{1} & R_{2} \\
R_{2}^{*} & R_{3}
\end{array}\right]=R
$$

Proof 5: Since for any matrix $M \in \kappa_{G}^{2 n \times 2 n}, \operatorname{in}_{+}(M)=\mathrm{in}_{-}(M)=n$, and there exist a matrix $E_{i i}=\left[\begin{array}{cc}I & 0 \\ 0 & I\end{array}\right]$, such that

$$
E_{i i} M E_{i i}^{*}=\left[\begin{array}{cc}
I_{n} & 0 \\
0 & -I_{n}
\end{array}\right]
$$

besides,

$$
N=\left(S_{1}-R_{1}^{-1}\right)^{-1} \in \kappa_{G}^{2 n \times 2 n}
$$

By similar argument, choose $S_{3} \in \kappa_{G}^{2 n \times 2 n}$, and $\operatorname{in}\left(S_{3}\right)=\operatorname{in}(N)$, then there exist $S_{2}$,

$$
N^{-1}=S_{2} S_{3}^{-1} S_{2}^{*}
$$

and $R_{2}, R_{3}$ can be easily determined.

\section{REFERENCES}

[1] C. Langbort, R. S. Chandra, and R. D'Andrea, "Distributed control design for systems interconnected over an arbitray graph," IEEE Transaction on Automatic Control, vol. 49, no. 9, pp. 1502-1519, Sep 2004.

[2] R. Andrea and G. Dullerud, "Distributed control design for spatially interconnected systems," IEEE Transaction on Automatic Control, vol. 48, no. 9, pp. 1478-1485, Sep 2003.

[3] G. Dullerud and R. D'Andrea, "Distributed control of heterogeneous systems," IEEE Transaction on Automatic Control, vol. 49, no. 12, pp. 2113-2128, Dec 2004.

[4] C. Langbort, V. Gupta, and R. Murray, "Distributed control over failing channels," in Lecture Notes in Control and Information Sciences series, P. J. Antsaklis and P. Tabuada, Eds., Workshop on Networked Embedded Sensing and Control, University of Notre Dame. Springer, 2006, pp. 325-342.

[5] C. Langbort and R. D'Andrea, "Distributed control of heterogeneous systems interconnected over an arbitrary graph," in Proceedings of the IEEE Conference on Decision and Control, 2003, pp. 2835-2840.

[6] V. A. Ugrinovskii, I. Peterson, A. Savkin, and E. Ugrinovskaya, "Decentralized state-feedback stabilization and robust control of uncertain large-scale systems with integrally constrained interconnections," System and Control Letters, vol. 40, no. 2, pp. 107-119, Jun 2000.

[7] G. Scorletti and G. Due, “An lmi approach to decentralized control," International Journal of Control, vol. 74, no. 3, pp. 211-224, Feb 2001.

[8] B. Chen and S. Lall, "Dissipation inequlaities for distributed systems on graph," in Proceedings of the IEEE Conference on Decision and Control, 2003, pp. 3084-3090.

[9] N. Motee. and A. Jadbabaie, "Receding horizon control of spatially distributed systems over arbitrary graphs," in Proceedings of the 45th IEEE Conference on Decision and Control, San Diego, CA, USA., Dec 2006, pp. 3467-3472.

[10] S. Tatikonda, "Control under communcation constraints," Ph.D. dissertation, Mass. Inst. Technol., Cambridge, MA, 2000.

[11] G. Nair and R. Evens, "Stabilization with data-rate limited feedback: Tightest attainable bounds," System and Control Letters, vol. 41, pp. 49-76, 2000.

[12] P. J. Antsaklis and J. Baillieul, "Guest editorial: Special issue on networked control systems," IEEE Transaction on Automatic Control, vol. 49, no. 9, pp. 1241-1243, Sep 2004.

[13] _ _ "Special issue on networked control systems technology," Proceedings of the IEEE, Jan 2007.

[14] Z. Di, M. Farhood, and G. E. Dullerud, "Control of distributed systems over graphs," in Proceedings of the 45th IEEE Conference on Decision and Control, San Diego, CA, USA, Dec 2006, pp. 3486-3491. 
[15] J. Willems, "Dissipative dynamical systems i \& ii," Arch. Rational Mech. Anal, vol. 45, pp. 321-393, 1972.

[16] A. Megretski and A. Rantzer, "System analysis via intergral qudratic constraints," IEEE Transaction on Automatic control, vol. 42, no. 6, pp. 819-830, Jun 1997.

[17] M. Cantoni, E. Weyer, Y. Li, S. Ooi, I. Mareels, and M. Ryan, "Control of large-scale irrigation networks." in Proceedings of IEEE, J. Bailliuel and P. Antsaklis, Eds., January 2007.

[18] A. Packard, "Gain scheduling via linear fractional transformations," System and Control letters, vol. 2, no. 22, pp. 79-92, Feb 1994.

[19] G. Scorletti and L. E. Ghaoui, "Improved linear matrix inequlity conditions for gain scheduling," International Journal of Robust and Nonlinear Control, vol. 8, no. 10, pp. 3626-3631, Aug 1998.

[20] M. Safonov, Stability and Robustness of Multivariable Feedback Systems. Cambridge, MA: MIT Press, 1980.

[21] P. Moylan and D. Hill, "Stability criteria for large-scale systems," IEEE Transaction on Automatic Control, vol. 23, no. 2, pp. 143-149, April 1978.

[22] T. Iwasaki and S. Hara, "Well-poseness of feedback systems: isights into exact robustness analysis and approximate computations," IEEE Transaction on Automatic Control, vol. 43, no. 5, pp. 619-630, May 1998.

[23] A. Helmersson, "Iqc synthesis based on inertial constraints," in Proceedings of the 14th IFAC World Congress, Beijing, China, 1999, pp. 163-168.

[24] C. Scherer, "Lpv control and full block multipliers," Automatica, vol. 37, no. 3, pp. 361-375, Mar 2001.

[25] G. Meinsma, T. Iwasaki, and M. Fu, "When is (d, g)-scaling both necessary and sufficient," IEEE Transaction on Automatic Control, vol. 45, no. 9, pp. 1755-1759, Sep. 2000

[26] J. Shamma, "Robust stability with time-varying strctured uncertainty," IEEE Transaction on Automatic Control, vol. 39, no. 4, pp. 714-724, April 1994.

[27] A. Megretski and S. Treil, "Power distribution inequalities in optimization and robustness of uncertain systems," Journal of Mathematical Systems, Estimation, and Control, vol. 3, no. 3, pp. 301-319, March 1993. 